\title{
Evaluation of Reliability and Validity of PDQ-39: Questionnaire in Iranian Patients With Parkinson's Disease
}

\author{
Azizallah Dehghan, ${ }^{1}$ Haleh Ghaem, ${ }^{2}$ Afshin Borhani-Haghighi, ${ }^{3}$ Roya Safari-Faramani, ${ }^{4,}$ Mahmood \\ Moosazadeh, ${ }^{5}$ and Ali Gholami ${ }^{6}$ \\ ${ }^{1}$ Noncommunicable Diseases Research Center, Fasa university of Medical Sciences, Fasa, IR Iran \\ ${ }^{2}$ Department of Epidemiology, Faculty of Health, Shiraz University of Medical Sciences, Shiraz, IR Iran \\ ${ }^{3}$ Faculty of Medicine, Shiraz University of Medical Sciences, Shiraz, IR Iran \\ ${ }^{4}$ Research Center for Modeling in Health, Institute for Futures Studies in Health, Kerman University of Medical Sciences, Kerman, IR Iran \\ ${ }^{5}$ Health Sciences Research Center, Faculty of Health, Mazandaran University of Medical Sciences, Sari, IR Iran \\ ${ }^{6}$ Department of Epidemiology, Faculty of Health, Iran University of Medical Sciences, Tehran, IR Iran \\ "Corresponding author: Roya Safari-Faramani, Research Center for Modeling in Health, Institute for Futures Studies in Health, Kerman University of Medical Sciences, Kerman, \\ IR Iran. E-mail: r.safari84@gmail.com
}

Received 2015 January 5; Accepted 2015 February 24.

\begin{abstract}
Background: Parkinson's disease is a progressive neurological disease. Measuring quality of life in these patients could be a useful tool for evaluation and prioritization of care services. The 39-item Parkinson's Disease questionnaire (PDQ-39) is an acceptable measure to assess quality of life in these patients.

Objectives: This study aims to assess the validity and reliability of the Persian version of PDQ-39 questionnaire.

Patients and Methods: In this cross-sectional study, Persian version of questionnaire was prepared with translation, back translation, cognation debriefing and culture adaptation. One hundred four Parkinson's disease patients referred to Shahid Motahari clinic affiliated to Shiraz University of Medical Sciences were included in the study. Then PDQ-39 and general quality of life questionnaire (36-Item short form health survey: SF-36) were completed by trained interviewers. The reliability of the questionnaire was measured through Cronbach's alpha coefficient. Construct validity assessed by item-scale correlation method and criterion validity was determined by discriminant validity and convergenent validity methods.

Results: Range of Cronbach's alpha coefficient for different dimensions varied from 0.64 to 0.92. Cronbach's alpha coefficient was higher than 0.70. Item-convergent validity ranged from 0.38 to 0.83 . Similar dimensions of PDQ-39 and SF-36 questionnaire were highly correlated. The quality of life among higher stage patients was lower $(\mathrm{P}<0.001)$.

Conclusions: Findings showed reliability and validity of Persian version of PDQ-39 is acceptable. It can be used to measure health related quality of life in Persian-speaking Parkinson's disease patients.
\end{abstract}

Keywords: Parkinson, Validity, Reliability, Quality of Life

\section{Background}

Parkinson's disease (PD) is a neurodegenerative disorder, characterized by rigidity, tremor, bradykinesia, masklike face and abnormal posture. It usually begins by the ages of 50 - 65 years and causes neuronal dysfunction. The prevalence is estimated to be 100 to 200 cases per 100,000 and the annual incidence is 20 cases per 100,000 $[1,2]$. Quality of life (QoL) is a wide spreading concept which encompasses all dimensions of life including health. It has physical, physiological, social and spiritual dimensions [3]. According to the definition of WHO it is "individuals" perception of their position in life in the context of the culture and value systems in which they live and in relation to their goals, expectations, standards and concerns" [4].

Usually, it is called "health-related quality of life" because of its close association with health [5]. In the cases of chronic disease, where the aim of treatment is to increase the level of satisfaction and improve QoL of patients rather than increase survival chance, the use of standard instruments for measuring QoL seems inevitable [5]. The PDQ39 is a widely used questionnaire with 39 questions measuring the effects of Parkinson's disease on QoL [6]. This questionnaire has been translated into several languages and utilized to measure QoL of patients with Parkinson's disease [7-12]. To be useful in clinical research applications, tools for measuring quality of life, as well as other scientific measuring instruments must be relevant, measurable, reliable and valid [13].

\section{Objectives}

As far as the PDQ-39 is concerned, it has not been standardized in the Iranian context. Thus, this study aims to

Copyright (c) 2016, Zahedan University of Medical Sciences. This is an open-access article distributed under the terms of the Creative Commons Attribution-NonCommercial 4.0 International License (http://creativecommons.org/licenses/by-nc/4.0/) which permits copy and redistribute the material just in noncommercial usages, provided the original work is properly cited. 
investigate the reliability and validity of the Persian duplicate of the PDQ-39.

\section{Patients and Methods}

\subsection{Translation and Adaptation}

In this cross-sectional study, the English version of the PDQ-39 was translated into Persian by a neurologist, an epidemiologist and a linguist. The Persian translation was in turn translated back into English by a different team including a neurologist, a nurse, and a linguist. After cultural adaptation, 25 patients completed the questionnaire to check comprehensibility, leading to the final Persian edition.

\subsection{Sample Size}

According to Fayers theory, in order to assess the validity and reliability of QoL questionnaires 100 to 400 patients are required [14].

\subsection{Patients}

From December 2010 to July 2011 all patients with Parkinson's disease referred to Shahid Motahari specialist clinic affiliated to Shiraz University of Medical Sciences, included in study. Two patients were excluded because of comorbidities. Both of them suffered stroke and were disable. Finally 104 patients were included. All patients singed an informed consent, initially. Patients were examined by a neurologist and using the Hohen and Yahr index, the stage of the disease was determined. Afterward, the Persian version of the PDQ-39 and SF-36 (a questionnaire designed to assess the general QoL) were completed by patients assisted by a nurse. The questionnaire filled by interview for illiterate patients and patients with severe tremor.

\subsection{Instrumrnts}

\subsubsection{Hohen and Yahr Index}

The $\mathrm{H}$ and $\mathrm{Y}$ index is an acceptable indicator of the severity of Parkinson's. It consists of five stages, with stage 1 (the least severe) and stage 5 (the most severe) [15].

SF-36 questionnaire is a general tool to assess quality of life. It was designed by Ware et al. consisting of eight dimensions: physical functioning, role limitations due to physical health, bodily pain, general health perceptions, vitality, social functioning, role limitations due to emotional health, and general mental health measures [16].

\subsubsection{Parkinson Disease Questionnaire-39 (PDQ-39)}

The PDQ-39 is a widely used questionnaire with 39 questions measuring the effects of Parkinson's disease on QoL under 8 dimensions including mobility (10 questions), activities of daily living ( 6 questions), emotional wellbeing (6 questions), stigma (4 questions), social support (3 questions), cognitive impairment (4 questions), communication (3 items) and bodily discomfort (3 questions) [6].

\subsection{Validity, Reliability and Statistical Analysis}

The internal consistency of this instrument was evaluated using Cronbach's alpha coefficient. Cronbach's alpha values fall between zero and one and the closer to 1 the higher the reliability. Also, values higher than 0.7 are defined as acceptable and those were higher than 0.8 are excellent [17]. The convergent validity of the two measures was assessed using Spearman's correlation coefficient. The values between 0 and 0.25 indicate a very weak correlation or no correlation at all; those between 0.25 and 0.50 indicate a weak correlation; those from 0.50 and 0.75 show good and medium correlations; and values of 0.75 and above are excellent [18]. In this study, the assumption was that the following pairs would be significantly correlated. Mobility (PDQ-39) and physical function (SF-36); activity of daily living (PDQ-39) and physical role(SF-36); wellbeing (PDQ-39) and mental health (SF-36); social support (PDQ-39) and social function (SF-36); and bodily discomfort (PDQ-39) and bodily pain (SF-36). The discriminant validity was calculated by comparing the $\mathrm{H}$ and Y-based scores of QoL at different stages of the disease and using the KruskalWallis test. The hypothesis here was that the questionnaire must discriminate the scores at different stages of the disease.

Construct validity was determined by item-convergent validity and item-discriminant validity. Spearman's correlation coefficient was used to evaluate item-convergent validity, with a value equal or above 0.4 considered acceptable. Item-discriminant validity was present when an item had a significantly higher correlation with its own dimension, compared with the other dimensions [19]. All statistical evaluations were made assuming a two-sided test based at 0.05 level of significance using SPSS-16.

\section{Results}

A total of 104 patients contributed to the analysis. The mean \pm SD age of participants was $65.0 \pm 11.6$ years with a male predominance (60.6\%). According to $\mathrm{H}$ and $\mathrm{Y}$ criterion 17 patients $(16.3 \%)$ in stage I, 40 (38.5\%) in stage II, 33 (30.8\%) in stage III, 13 (12.5\%) in IV and two were in stage $\mathrm{V}$. The demographic and clinical characteristics of participants are presented in Table 1 . 
Table 1. Demographic and Clinical Characteristics of Study Participants ${ }^{\mathrm{a}}$

\begin{tabular}{|c|c|}
\hline Variable & Value \\
\hline Age, $y$ & $65.00 \pm 11.6$ \\
\hline Onset of disease & $60.67 \pm 12.1$ \\
\hline Disease duration & $4.19 \pm 4.3$ \\
\hline$H$ and $Y$ stage & $2.4 \pm 0.9$ \\
\hline \multicolumn{2}{|l|}{ Sex, No. (\%) } \\
\hline Male & $63(60.6)$ \\
\hline Female & $41(39.4)$ \\
\hline \multicolumn{2}{|c|}{ Marital status, No. (\%) } \\
\hline Single & $3(2.9)$ \\
\hline Married & $81(77.9)$ \\
\hline Separation & $2(1.9)$ \\
\hline Widowed & $18(17.3)$ \\
\hline
\end{tabular}

${ }^{\mathrm{a}}$ Values are expressed as mean \pm SD unless otherwise indicated.

Table 2 shows the value of Coronbach's $\alpha$ coefficients and Mean \pm SD of quality of life score for different dimensions. The patients had most problem in mobility dimension (highest score) and the lowest in social support dimensions (lowest score). Except for stigma dimension, Coronbach's $\alpha$ coefficients were higher than 0.7 for other dimensions.

Item-convergent validity and item-discriminant validity are presented in Table 3. For item-convergent validity all coefficients were more than 0.4 except in one item. The scores of PDQ-39's dimensions among persons at different $\mathrm{H}$ and $\mathrm{Y}$ stages were shown in Table 4.

The quality of life score was positively associated with the stage of disease. That is patients with the higher stage had lower QoL score. Convergent validity assessed by calculating spearman correlation coefficient between different dimensions of PDQ-39 and SF-36. There were acceptable correlations between the same dimensions of two questionnaires. The mobility, activity of daily living, emotional well-being, social support and bodily discomfort of PDQ-39 questionnaire were considered as equal to physical function, physical role, mental health, social function and bodily pain dimensions of the SF-36 questionnaire respectively.

\section{Discussion}

In order to be beneficial in clinical research, an instrument requires acceptable reliability and validity. In this study, found that the Persian version of the PDQ-39 had a very good internal consistency. Cronbach's alpha coefficient varied between 0.64 and 0.92 except for stigma di- mension (Cronbach's alpha $=0.64)$. This may be due cultural probsems and adapting appropriate coping strategies. It was similar to Peto et al.'s study on the original questionnaire [20].

Cronbach's alpha coefficient for physical discomfort and social support dimensions were 0.71 and 0.74 , respectively which were acceptable. Findings were similar to the other studies carried out in other parts of the world. Cronbach's correlation coefficients for some other countries varied between 0.69 to 0.94 in South Korea [7], 0.63 to 0.94 in Spain [9], 0.69 to 0.94 in UK [20], 0.48 to 0.91 again in China [21], 0.64 to 0.90 in Singapore [8] and 0.51 to 0.96 in the USA [12]. Comparing to these studies, the coefficients obtained are acceptable.

There were acceptable correlation between 38 out of 39 questions and their related dimensions (Table 3 ). Also the correlation between each of the questions and the other dimensions was lower than the correlation between their related ones. These demonstrate that each question is able to measure its dimensions and also to discriminate the other ones. The correlation was less than 0.4 in the case of question numbered 25 in stigma dimension.

It seems that this is due to cultural maladjustment. May be it is because of different concept and viewpoints in Iranian patients.

According to the result of the present study the higher stage of Parkinson's the higher the score of QoL reflected the lower QoL. The mean of scores on all dimensions of the questionnaire is higher at higher stages, which certainly indicates the lower QoL in patients with more severe stages of Parkinson's. This finding sheds light on the discrimi- 
Table 2. Mean and Cronbach's $\alpha$-Coefficients for Each Dimensions of PDQ-39 Questionnaire ( $=104)$

\begin{tabular}{lll}
\hline Dimensions & Mean \pm SD & Cronbach's alpha \\
\hline Mobility & $57.55 \pm 17.0$ & 0.92 \\
\hline Activities of daily living & $34.63 \pm 21.0$ & 0.88 \\
\hline Emotional well-being & $32.04 \pm 16.3$ & 0.85 \\
\hline Stigma & $48.61 \pm 20.9$ & 0.64 \\
\hline Social support & $24.51 \pm 19.4$ & 0.74 \\
\hline Cognition & $33.81 \pm 18.2$ & 0.84 \\
\hline Communication & $41.83 \pm 17.8$ & 0.82 \\
\hline Bodily discomfort & $38.41 \pm 19.1$ & 0.71 \\
\hline PDQ-39 summary index & $44.81 \pm 15.6$ \\
\hline
\end{tabular}

Table 3. Item Scaling Tests: Convergent and Discriminant Validity for PDQ-39 Dimensions

\begin{tabular}{lccc}
\hline Dimensions & Number of Items & Item-Convergent Validity (Range of Correlation) & Item-Discriminant Validity (Range of Correlation) \\
\hline Mobility & 10 & $0.49-0.81$ & $0.27-0.51$ \\
\hline Activity of daily living & 6 & $0.56-0.83$ & $0.25-0.49$ \\
\hline Emotional well-being & 6 & $0.65-0.82$ & $0.20-0.38$ \\
\hline Stigma & 4 & $0.38-0.62$ & $0.22-0.46$ \\
\hline Social support & 3 & $0.58-0.79$ & $0.29-0.50$ \\
\hline Cognition & 4 & $0.52-0.68$ & $0.31-0.47$ \\
\hline Communication & 3 & $0.58-0.72$ & $0.23-0.41$ \\
\hline Bodily discomfort & 3 & $0.44-0.59$ & $0.26-0.34$ \\
\hline
\end{tabular}

Table 4. Mean and Significance Level of Dimensions of PDQ-39 at Different Stages of Disease ${ }^{\mathrm{a}}$

\begin{tabular}{|c|c|c|c|c|c|}
\hline \multirow[t]{2}{*}{ Dimensions } & \multicolumn{4}{|c|}{ Hohen and Yahr Scale } & \multirow[t]{2}{*}{ P Value } \\
\hline & Stage $1(N=17)$ & Stage $2(N=40)$ & Stage $3(N=32)$ & Stage $4,5(N=15)$ & \\
\hline Mobility & $24.00 \pm 15.0$ & $28.00 \pm 11.4$ & $42.75 \pm 14.4$ & $55.76 \pm 11.4$ & 0.0001 \\
\hline Activities of daily living & $16.44 \pm 9.0$ & $31.56 \pm 16.3$ & $42.75 \pm 14.4$ & $62.15 \pm 17.4$ & 0.0001 \\
\hline Emotional well-being & $31.77 \pm 11.3$ & $39.37 \pm 12.1$ & $50.08 \pm 15.0$ & $60.98 \pm 14.7$ & 0.0001 \\
\hline Stigma & $25.00 \pm 16.0$ & $41.09 \pm 15.3$ & $50.00 \pm 15.4$ & $67.94 \pm 11.0$ & 0.0001 \\
\hline Social support & $28.88 \pm 11.8$ & $38.75 \pm 15.3$ & $54.50 \pm 19.6$ & $64.70 \pm 11.7$ & 0.0001 \\
\hline Cognition & $28.33 \pm 10.9$ & $40.46 \pm 14.5$ & $55.50 \pm 17.4$ & $65.00 \pm 11.0$ & 0.0001 \\
\hline Communication & $40.47 \pm 12.1$ & $44.79 \pm 15.3$ & $57.87 \pm 13.9$ & $66.27 \pm 17.3$ & 0.0001 \\
\hline Bodily discomfort & $31.11 \pm 20.1$ & $37.91 \pm 16.9$ & $57.43 \pm 15.3$ & $58.64 \pm 16.8$ & 0.0001 \\
\hline PDQ-39 summary index & $27.28 \pm 10.3$ & $35.80 \pm 9.8$ & $53.00 \pm 15.6$ & $61.38 \pm 11.3$ & 0.0001 \\
\hline
\end{tabular}

${ }^{\mathrm{a}}$ Values are expressed as mean $\pm \mathrm{SD}$.

nant power of the PDQ-39. These results can be compared to a study by Zhang et al. in China [21]. The scores of the questionnaire's summary and the dimensions of mobility and activities of daily living were significantly different. In another study in China by Luo et al. the correlation was significant only in relation with mobility, activities of daily living, emotional health and social support [22]. However, in our study the relationship was significantly different in 
all aspects. In Martinez-Martin and Frades Payo's study in Spain [9], there was a significant difference between the score on QoL in all dimensions and the score on the PDQ36 's summary at the disease's stages based on the $\mathrm{H}$ and $\mathrm{Y}$ index.

Dimensions of mobility, activities of daily living, emotional wellbeing, social support, and physical discomfort in the PDQ-39 correlated to an acceptable extent to the dimensions of physical function, physical role, mental health, social functioning, and bodily pain, respectively, in the SF-36. Despite these, some dimensions of the former, namely cognitions, stigma and cognitions bore no significant correlation with any dimension in the latter (Table 5). Correlation coefficients were all negative as the higher scores of the PDQ-39 and the lower scores of the SF-36 signify lower QoL. The results of the current study are consistent with those of Luo et al.'s [22] and Zhang and Chan's in China [21].

In conclusion, the results of this study demonstrated that the validity and reliability of the Persian version of the PDQ-39 are acceptable, and it can thus be recommended as a self-reported questionnaire for measuring the QoL of patients with Parkinson's disease. We hope that this instrument can be easily used by researchers and clinicians to better evaluate the QoL of patients with Parkinson. Thereby enhancing their health status and wellbeing the authors would be glad to provide the enthusiasts with this instrument without charge.

\section{Acknowledgments}

This is the result of thesis for Master of Sciences degree in Epidemiology approved in Shiraz University of Medical Sciences numbered AD7462 by Azizallah Dehghan which was performed in Shahid Motahari Clinic in Shiraz, Iran. We should appreciate kind co-operation of the staff of Shahid Motahari Clinic and all translator.

\section{Footnotes}

Authors' Contribution: All the authors have made substantial contributions to Azizallah Dehghan and Haleh Ghaem contributed in the conception and design of the study. Azizallah Dehghan, Afshin Borhani-Haghighi and Ali Gholami contributed in data acquisition. Azizallah Dehghan, Roya Safari-Faramani and Mahmood Moosazadeh contributed in data analysis and interpretation drafting of the article and its revision.

Funding/Support: Shiraz University of Medical Sciences. 
Table 5. Spearman's Rank Correlation Between PDQ-39 and SF-36 Dimensions

\begin{tabular}{|c|c|c|c|c|c|c|c|c|}
\hline \multirow{2}{*}{$\begin{array}{l}\text { PDQ-39 } \\
\text { dimensions }\end{array}$} & \multicolumn{8}{|c|}{ SF-36 Dimension } \\
\hline & $\begin{array}{l}\text { Physical } \\
\text { Function }\end{array}$ & Role-Physical & Bodily Pain & General Health & $\begin{array}{c}\text { Social } \\
\text { Function }\end{array}$ & Vitality & $\begin{array}{c}\text { Role- } \\
\text { Emotional }\end{array}$ & Mental Health \\
\hline Mobility & $-0.72^{\mathrm{a}}$ & $-0.54^{\mathrm{a}}$ & -0.36 & $-0.40^{\mathrm{b}}$ & $-0.47^{\mathrm{b}}$ & -0.42 & -0.41 & -0.32 \\
\hline $\begin{array}{l}\text { Activities of } \\
\text { daily living }\end{array}$ & $-0.52^{\mathrm{a}}$ & $-0.69^{a}$ & -0.18 & -0.26 & $-0.55^{\mathrm{a}}$ & -0.51 & -0.37 & -0.30 \\
\hline $\begin{array}{l}\text { Emotional } \\
\text { well-being }\end{array}$ & -0.22 & $-0.36^{b}$ & -0.28 & -0.25 & $-0.46^{\mathrm{a}}$ & -0.68 & -0.33 & $-0.68^{a}$ \\
\hline Stigma & -0.28 & -0.29 & -0.24 & -0.18 & $-0.45^{\mathrm{b}}$ & $-0.38^{b}$ & -0.22 & -0.32 \\
\hline Social support & $-0.30^{\mathrm{b}}$ & -0.27 & $-0.35^{\mathrm{b}}$ & -0.27 & $-0.71^{a}$ & $-0.51^{a}$ & $-0.39^{b}$ & $-0.36^{b}$ \\
\hline Cognition & $-0.33^{\mathrm{b}}$ & $-0.32^{\mathrm{b}}$ & -0.21 & -0.27 & $-0.42^{\mathrm{b}}$ & -0.43 & -0.24 & -0.21 \\
\hline Communication & $-0.41^{\mathrm{b}}$ & $-0.31^{b}$ & -0.15 & -0.34 & $-0.56^{\mathrm{a}}$ & $-0.44^{\mathrm{a}}$ & -0.35 & $-0.38^{\mathrm{a}}$ \\
\hline $\begin{array}{l}\text { Bodily } \\
\text { discomfort }\end{array}$ & $-0.46^{\mathrm{b}}$ & $-0.32^{b}$ & $-0.67^{\mathrm{a}}$ & $-0.37^{b}$ & -0.34 & $-0.38^{b}$ & -0.13 & -0.16 \\
\hline $\begin{array}{l}\text { PDQ-39 } \\
\text { summary } \\
\text { index }\end{array}$ & $-0.59^{\mathrm{a}}$ & $-0.56^{\mathrm{a}}$ & $-0.39^{b}$ & $-0.45^{b}$ & $-0.68^{a}$ & $-0.47^{\mathrm{b}}$ & $-0.42^{\mathrm{b}}$ & $-0.46^{\mathrm{a}}$ \\
\hline
\end{tabular}

\section{References}

1. Amino MJ. Parkinson's disease and other extrapyra-midal disorders In: Ongo D, Fauci A, Kasper D, editors. Harrison's principles of internal medicine. New York: McGraw Hill; 2011. .

2. Yahr MD. Parkinsonism. In: Rowland LP, Pedley TA, editors. Meritt's neurology. 12 ed. Washington D.C: LWW Press; 2009. .

3. Guyatt GH, Feeny DH, Patrick DL. Measuring health-related quality of life. Ann Intern Med. 1993;118(8):622-9. [PubMed: 8452328].

4. Ghaem H, Borhani-Haghighi A, Zeighami B, Dehghan A. Validity and reliability of the Persian version of the Parkinson Disease Quality of Life (PDQL) questionnaire [in Persian]. J Kerman Univ Med Sci. 2010;17(1):49-58.

5. Ghaem H, Borhani-Haghighi A. Validity and reliability of the Persian epilepsy quality of life questionnaire. Neurosciences (Riyadh). 2010;15(4):249-53. [PubMed: 20956921].

6. Jenkinson C, Fitzpatrick R, Peto V, Greenhall R, Hyman N. The Parkinson's Disease Questionnaire (PDQ-39): development and validation of a Parkinson's disease summary index score. Age Ageing. 1997;26(5):353-7. [PubMed: 9351479].

7. Park HJ, Sohng KY, Kim S. Validation of the Korean version of the 39-Item Parkinson's Disease Questionnaire (PDQ-39). Asian Nurs Res (Korean Soc Nurs Sci). 2014;8(1):67-74. doi: 10.1016/j.anr.2014.02.004. [PubMed: 25030495].

8. Tan LC, Luo N, Nazri M, Li SC, Thumboo J. Validity and reliability of the PDQ-39 and the PDQ-8 in English-speaking Parkinson's disease patients in Singapore. Parkinsonism Relat Disord. 2004;10(8):493-9. doi: 10.1016/j.parkreldis.2004.05.007. [PubMed:15542010].

9. Martinez-Martin P, Frades Payo B. Quality of life in Parkinson's disease: validation study of the PDQ-39 Spanish version. The Grupo Centro for Study of Movement Disorders. J Neurol. 1998;245 Suppl 1:S34-8. [PubMed: 9617722].

10. Luo N, Tan LC, Li SC, Soh LK, Thumboo J. Validity and reliability of the Chinese(Singapore) version of the Parkinson's Disease Questionnaire (PDQ-39). Qual Life Res. 2005;14(1):273-9. [PubMed: 15789961].
11. Krikmann U, Taba P, Lai T, Asser T. Validation of an Estonian version of the Parkinson's Disease Questionnaire (PDQ-39). Health Qual Life Outcomes. 2008;6:23. doi: 10.1186/1477-7525-6-23. [PubMed: 18364047].

12. Bushnell DM, Martin ML. Quality of life and Parkinson's disease: translation and validation of the US Parkinson's Disease Questionnaire (PDQ-39). Qual Life Res. 1999;8(4):345-50. [PubMed: 10472167].

13. Jaeschke R, Guyatt GH. How to develop and validate a new quality of life instrument. In: Spilker B, editor. Quality of life assessments in clinical trials. New York: Raven Press; 1990. pp. 47-57.

14. Fayers PM, Machin D. Scores and Measurements: Validity, Reliability, Sensitivity. In: Fayers PM, Machin D, editors. Quality of life: The assessment, analysis, and interpretation of patient-reported outcomes.. 2 ed. New York: Wiley Press; 2007. pp. 43-71.

15. Dehghan AA, Ghaem H, Borhani-Haghighi A, Kashfi SM, Zeyghami B Comparison of quality of life in Parkinson's patients with and without fatigue [in Persian]. Med J Hormozgan Univ. 2011;15(1):49-55.

16. Ware JE, Snow KK, Kosinski M. SF-36 health survey: Manual and in terpretation guide. New England: The Health Institute, New England Medical Center; 1993.

17. Aday LA, Cornelius LJ. Designing and conducting health surveys: A comprehensive guide. 3 ed. Oakland: Jossey-Bass Press; 2006

18. Portney LG, Watkins MP. Foundations of clinical research: Applications to practice. 3 ed. USA: Prentice Hall Press; 2008.

19. Fayers PM, Machin D. Quality of life: Assessment analysis and interpretation. New York: Wiley Press; 2000. pp. 72-9.

20. Peto V, Jenkinson C, Fitzpatrick R. PDQ-39: a review of the development, validation and application of a Parkinson's disease quality of life questionnaire and its associated measures. J Neurol. 1998;245 Suppl 1:S10-4. [PubMed: 9617716].

21. Zhang JL, Chan P. Reliability and validity of PDQ-39: a quality-of-life measure for patients with PD in China. Qual Life Res. 2012;21(7):121721. doi: 10.1007/s11136-011-0026-1. [PubMed: 21983714].

22. Luo W, Gui XH, Wang B, Zhang WY, Ouyang ZY, Guo Y, et al. Validity and reliability testing of the Chinese (mainland) version of the 39item Parkinson's Disease Questionnaire (PDQ-39). J Zhejiang Univ Sci B. 2010;11(7):531-8. doi: 10.1631/jzus.B0900380. [PubMed: 20593519] 